\title{
Alcances y problemas del dialeteísmo
}

Resumen. El dialeteísmo es la posición que afirma que hay contradicciones verdaderas. Este artículo versará sobre esa posición. En la primera sección, mencionaré los principales aportes que, en mi perspectiva, el dialeteísmo ha hecho a la lógica filosófica. En la segunda sección, analizaré el principal problema del dialeteísmo. En la tercera sección, mostraré que los argumentos a favor del dialeteísmo no llegan a establecer la verdad de esta posición. Finalmente, explicaré cuál es el tipo de paraconsistencia que considero adecuada y la compararé con la posición dialeteísta.

Palabras Clave: dialeteísmo, paraconsistencia, paradoja del mentiroso, sorites.

Abstract: Dialetheism is the position which claims that there are true contradictions. This paper deals with this position. In the first section, I will mention the main contributions that, in my perspective, dialetheism has made to philosophical logic. In the second section, I will analyze the main problem of dialetheism. In the third section, I will show that the arguments in favor of dialetheism are not conclusive. Finally, I will explain which kind of paraconsistency I consider adequate, and I will compare it with the dialetheist position.

Keywords: dialetheism, paraconsistency, liar paradox, sorites

\section{Dialeteísmo y anti-dogmatismo}

Uno de los prejuicios más comunes sobre la lógica es que se trata de una disciplina que se apoya sobre ciertos dogmas incuestionables: el tercero excluido y la no-contradicción, particularmente. El primero de estos principios había sido ya discutido por Aristóteles, y desafiado por un interesante conjunto de matemáticos y lógicos (entre otros, Lukasiewicz y los intuicionistas). Sin embargo, la no-contradicción solía ser mantenida como la piedra basal de la lógica, y no era posible modificar ese principio central.

\footnotetext{
* Licenciado en Filosofía por la Universidad de Buenos Aires, actualmente realizando el doctorado en la misma universidad, financiado con una beca doctoral del CONICET. Sus temas de investigación son filosofía de la lógica y epistemología formal. Dirección electrónica: diegotajer@gmail.com
} 
La lógica paraconsistente fue un primer paso al respecto: ya en los años 50' se comenzaron a desarrollar lógicas que rechazaban el Ex falso por diferentes razones (este rechazo es lo que las define como paraconsistentes). La principal razón, sin embargo, era la anti-intuitividad de inferir de una contradicción cualquier otra afirmación. Pero el principio de no contradicción, que establece que no hay contradicciones verdaderas, permanecía incólumne.

Fue particularmente gracias a Graham Priest que este principio comenzó a ser discutido entre la comunidad de lógicos. Según este autor, hay contradicciones verdaderas. Entre otras oraciones verdaderas y falsas, se encuentran la paradójica oración del Mentiroso ('esta oración es falsa'), y plausiblemente enunciados sobre estados de cambio ('esto es una copa', dicho en el instante en que la copa se está rompiendo) o de vaguedad. Como muchas otras lógicas no-clásicas, el dialeteísmo no puede ser una mera alternativa a la lógica clásica, sino que debe ser rival. El relevantismo, por ejemplo, no es necesariamente rival de la lógica clásica, justamente porque puede admitir que el Ex falso, aunque inválido, preserva verdad en mundos posibles. Por el contrario, para quien considere que hay contradicciones verdaderas, las inferencias clásicas como el Ex falso son vistas como inválidas porque no preservan verdad simpliciter, y por eso su posición no puede integrarse con la clásica.

El principal mérito del dialeteísmo es metodológico: llevó la posibilidad de revisión de la lógica más allá de su límite tradicional. Recordemos el locus clásico del revisionismo (Neurath 1932, p. 206): "Somos como navegantes que tienen que transformar su nave en pleno mar, sin jamás poder desmantelarla en un dique de carena y reconstruirla con los mejores materiales". Esta cita es comúnmente utilizada por los defensores del revisionismo. Veamos, sin embargo, cuál es el principio fundamental que guía la revisión para Neurath (1932, p. 205): "En la ciencia unitaria tratamos de crear un sistema libre de contradicción (...) Cuando se nos muestra una nueva proposición, la comparamos con el sistema de que disponemos, y averiguamos si la nueva proposición se halla o no en contradicción con el sistema". Vemos, entonces, que para un famoso holista como Neurath, la no-contradicción sigue siendo un principio central. Más contemporáneamente, el coherentismo, la escuela opuesta al fundacionalismo, supone en general un requisito de consistencia. 
De esta manera, el dialeteísmo logra cuestionar el principio más fundamental que había permanecido (generalmente) incuestionable por parte de la comunidad de lógicos hasta ese momento, incluso por parte de aquellos que sostenían que la revisión de la lógica era posible o conveniente. El dialeteísmo ha motivado una manera distinta de ver la lógica, que siempre estuvo asociada con la no-contradicción como un requisito al menos necesario. El punto de Priest fue que la lógica va mucho más allá de eso, y que los argumentos válidos no tienen por qué estar directamente relacionados con la preservación de consistencia. Asimismo, dio lugar para que el que postula la existencia de contradicciones verdaderas no sea por eso un anti-lógico o un irracionalista. El debate sobre el principio de no-contradicción no es entonces el debate entre un ser racional y uno irracional que hace críticas externas, sino un debate entre dos posiciones internamente coherentes y con lógicas propias.

\section{Dialeteísmo y dogmatismo}

El primer problema que rodea al dialeteísmo es quizás el más fuerte, aunque probablemente el más fácil de rebatir. Se trata de la consideración del principio de no-contradicción como algo que no puede abandonarse de ninguna manera. La mera existencia de contradicciones verdaderas hace temblar a la gran mayoría de los filósofos y lógicos, y el dialeteísmo es así rechazado antes de ser considerado como opción.

¿Debemos considerar que los defensores de la no-contradicción de este tipo son meros dogmatistas? Creo que la respuesta depende del grado en que estos filósofos permiten el diálogo con los dialeteístas. La no-contradicción es un principio central en varias ramas de la ciencia y la filosofía, y su defensa teórica data al menos desde la Metafísica de Aristóteles. No podemos considerarla como un mero prejuicio o una intuición preteórica infundada; tampoco podemos ver la consistencia como un requisito del mismo nivel que otros (categoricidad, simpleza, etc.) para una teoría lógica, que puede ser abandonado con cierta facilidad. Si la discusión ha de ponerse en un suelo firme, el dialeteísta debe admitir dos cosas: en primer lugar, el inconmensurable valor teórico que la no-contradicción posee en la ciencia y la filosofía contemporáneas; y en segundo lugar, la fuerza intuitiva, no sólo de este principio, sino también de otros principios inferenciales 
asociados (por ejemplo, el silogismo disyuntivo). ${ }^{1}$ De este modo, si han de darse verdaderas razones para aceptar la existencia de contradicciones verdaderas, éstas deben ser lo suficientemente fuertes como para abandonar un principio tan instalado en nuestras creencias y nuestras teorías.

El relativo dogmatismo respecto a la no-contradicción sostenido por los clásicos también puede basarse en la ausencia de una base común con los dialeteístas. En palabras de Lewis (1982, p. 434; la traducción es mía):

Ninguna verdad tiene, ni puede tener, una negación verdadera. Nada es, ni puede ser, literalmente verdadero y falso a la vez. (...) Esto puede parecer dogmático. Y lo es: estoy afirmando la misma tesis que Routley y Priest cuestionaron, y (contrario a las reglas del debate) rechacé defenderla. Más aún, considero que es indefendible contra su ataque. Ellos han puesto en cuestión tanto que no hay un lugar indisputado donde se pueda pisar firme. Bastante malo para la demanda de que los filósofos deban siempre estar dispuestos a defender sus tesis bajo las reglas del debate.

Sin embargo, esto es exagerado. Hay mucho terreno común entre el clásico y el dialeteísta (de hecho, las lógicas paraconsistentes suelen ser sublógicas de la lógica clásica), y la posibilidad de discusión existe y debe ser explorada. El mismo Lewis (2001), en una carta a Priest, expresó una postura más abierta, que expresa la actitud no dogmática que considero adecuada en este debate: "Paraconsistencia. Estoy cada vez más convencido de que puedo y de hecho razono sobre situaciones inconsistentes (Sylvan's Box me convenció en gran parte). Pero no entiendo realmente cómo es que eso funciona" (2001, p. 176; la traducción es mía).

Naturalmente, el debate no necesariamente es sólo de índole semántica sino también metafísica, pues el principio de no-contradicción es históricamente un principio metafísico, aunque esté contemporáneamente planteado en términos de oraciones contradictorias y no de hechos.

Algunas de las disciplinas donde el principio de no-contradicción está muy instalado tienen que ver con la racionalidad y el lenguaje. En las teorías de revisión de creencias, suele considerarse que la presencia de una

\footnotetext{
${ }^{1}$ Si hubiera contradicciones verdaderas, entonces no podríamos pasar de $\mathrm{AvB}$ y $\neg \mathrm{B}$ hacia A. Pues podría suceder que tanto B como $\neg$ B fueran verdaderas mientras que A fuera falsa, y en ese caso la regla no preservaría verdad.
} 
contradicción es condición suficiente para revisar un cuerpo de creencias y eliminar alguno de los factores que llevaban a ella (parecido a lo que Neurath proponía, en la sección anterior). Asimismo suele considerarse que la aserción de una oración implica el rechazo de su negación por parte del sujeto. Si alguien dice "hoy tengo turno con el dentista", parece estar dándonos información suficiente como para considerar que "hoy no tengo turno con el dentista" es algo que él no cree.

Desde ya, Priest tiene respuestas, al menos esquemáticas, para estos casos. Sostiene que tener creencias inconsistentes no necesariamente es síntoma de un problema (por ejemplo, alguien puede creer muy racionalmente que la oración del mentiroso es verdadera y falsa), y que puede elaborarse una teoría de la revisión de creencias que soporte inconsistencias. ${ }^{2} \mathrm{El}$ truco aquí consiste en separar dos cosas que la tradición une: el rechazo de una proposición y la aceptación de su negación. Según Priest, rechazar una proposición implica una actitud más fuerte que aceptar la negación de ella; y si bien no podemos aceptar y rechazar lo mismo, ${ }^{3}$ sí podemos aceptar una proposición y su negación. Sin embargo, la plausibilidad de esta propuesta no está garantizada por su coherencia interna: también implica un cambio bastante radical en nuestra concepción de lo que significa afirmar algo.

Para calmar el debate, algunos dialeteístas suponen que el abandono de la no-contradicción no es tan grave, pues los enunciados contradictorios y verdaderos son muy pocos y en la gran mayoría de las regiones discursivas se puede razonar clásicamente. Esta respuesta me parece filosóficamente irresponsable. El hecho de que exista una contradicción verdadera implica un cambio radical en nuestra concepción sobre la falsedad, la verdad y la consecuencia lógica (entre otras cosas), y la restricción de consistencia aplicada a dominios presuntamente consistentes es simplemente una manera de hacer feliz al que usa cotidianamente lógica clásica (por ejemplo, un matemático profesional), pero es en el fondo una estrategia que borra los límites entre lo formal y lo material, dejando a la no-contradicción en algún limbo intermedio, y tomándose poco en serio los nuevos principios que la lógica paraconsistente pone sobre la logicidad.

\footnotetext{
${ }^{2}$ Véase, por ejemplo, Priest (2006), capítulo 8.

${ }^{3}$ Para Grinn (2004, p. 62), desde cierta perspectiva esto implica que Priest mantiene la no-contradicción, sólo que la traslada desde un plano semántico a uno pragmático.
} 


\section{Argumentos a favor del dialeteísmo}

En esta sección, analizaré los principales argumentos a favor del dialeteísmo. Mi propósito no será dar respuestas u objeciones definitivas a estos argumentos (tarea que podría llevarme un libro entero), sino analizar en qué medida logran su objetivo de motivar la aceptación de contradicciones verdaderas. Al contrario del orden expositivo típico, desarrollaré en las primeras subsecciones las motivaciones no lógicas (es decir, las metafísicas y normativas), y finalmente pasaré a las motivaciones lógicas (sin duda, más fuertes y comúnmente citadas).

\subsection{Argumentos metafísicos: verdad y cambio}

Si bien Priest confiesa que al principio el dialeteísmo sólo apuntaba a problemas relativos a las paradojas semánticas (Priest 2006a, p. 159), actualmente el debate también se da en problemas metafísicos tradicionales. En algún punto esto vuelve más fuerte al problema de las contradicciones, pues aceptar que hay conceptos contradictorios (paradigmáticamente, el concepto de Verdad) es algo relativamente tolerable, pero aceptar que una oración contradictoria y puramente descriptiva sobre el mundo sea verdadera puede resultar excesivo.

Pensemos primero en las paradojas de vaguedad. Típicamente este tipo de argumento paradójico incluye una premisa que caracteriza el uso de los términos vagos: que si $A(n)$, entonces $A(n+1)$. Por ejemplo: si una película de 60 minutos es corta, una película de 61 minutos también lo será; si un perro de 20 días es cachorro, un perro de 21 días también lo es.

La estructura de estas paradojas es así:

1. P0 [p.ej. Un ser humano de cero meses es joven]

2. $\mathrm{P}(\mathrm{n}) \rightarrow \mathrm{P}(\mathrm{n}+1)$ [Si un ser humano de $\mathrm{n}$ meses es joven, entonces un ser humano de $n+1$ meses es joven]

3. $\square \mathrm{nP}(\mathrm{n})<$ Por Inducción matemática, entre (1) y (2) $>$ [Todo ser humano, de cualquier edad, es joven]

4. $\mathrm{P}(992)<$ Por instanciación de (3)> [Un ser humano de 992 meses (alrededor de 85 años) es joven]

De esta manera, a partir de la aceptación de (1) y (2), y principios comúnmente aceptados como la inducción matemática, llegamos a un 
enunciado flagrantemente falso como (4). ¿Qué hacer frente a esto? No parece tan fácil rechazar (1), y decir que un ser humano de cero meses no es joven, ni tampoco rechazar (2), lo que requeriría establecer un límite según el cual un ser humano de $\mathrm{n}$ meses es joven, pero uno de $\mathrm{n}+1$ meses no lo es. La primera opción equivaldría a decir que nadie es joven, convirtiendo a un concepto ordinario en un concepto ficcional o erróneo por sí mismo. La segunda opción, por otro lado, va en contra de nuestras intuiciones semánticas fundamentales respecto a los conceptos vagos.

Sin embargo, la mayoría de las respuestas a esta paradoja optan por abandonar (2), lo cual considero correcto. En el caso de la vaguedad es filosóficamente adecuado abandonar esta premisa, pues más allá de si somos dialeteístas, supervaluacionistas o clásicos, no es razonable aceptar la conclusión. Por más que (2) resulta verdaderamente intuitiva, esta paradoja muestra que debe ser falsa. Esto es distinto a lo que sucede en el caso de la paradoja del mentiroso, donde la conclusión (plausiblemente "la oración del Mentiroso es verdadera y falsa") no es tan flagrantemente inaceptable.

Hay dos respuestas tradicionales al problema de la vaguedad, que proponen abandonar (2): las gappy y las glutty. La primera postula que en casos intermedios, los predicados vagos no se aplican: por ejemplo, en cierto intervalo de edad, no podemos decir correctamente de alguien que sea joven o que no lo sea. ${ }^{4}$ Antes de ese intervalo, podemos considerar que era joven; y después, que no lo es. Las soluciones glutty, por otro lado, sostienen que en los casos intermedios se aplica y no se aplica el predicado. Es decir, que en el mismo intervalo de edad que antes consideré, las personas son jóvenes y no lo son.

Una manera de defender el dialeteísmo es a partir de las soluciones glutty a las paradojas de vaguedad. Sin embargo, considero que pueden hacerse varias observaciones a esta motivación para el dialeteísmo. En primer lugar, y esto podría afectar a ambas soluciones, el problema parece meramente trasladarse de lugar pero no solucionarse: pues ahora también es preciso postular un punto determinado en el que el joven empieza a caer en la categoría de "joven y no joven" (o "ni joven ni no-joven", en la versión $g a p p y)$. Sin embargo, había sido justamente la ausencia de tales

\footnotetext{
${ }^{4}$ Una variante de las propuestas gappy es el supervaluacionismo, donde no podemos decir que ese hombre sea joven ni tampoco que no lo es, pero sí podemos decir ese hombre es joven o no lo es.
} 
límites estrictos lo que nos había llevado a aceptar la premisa (2). Ahora estamos proponiendo límites conceptualmente distintos a los de antes (pues, por ejemplo, ya no son límites entre joven y no-joven, sino entre joven y joven-y-no-joven) pero igual de estrictos, disponiéndonos a pagar el precio adicional de aceptar gluts o gaps. Por esta razón, puede convenir la aceptación de límites estrictos desde un comienzo, evitando introducir gaps y gluts, y suponer, como le gustaría al clásico, que se da simplemente un cambio entre joven y no-joven en algún punto determinado, ${ }^{5}$ manteniendo nuestros principios clásicos.

En segundo lugar, y ya respecto de la solución dialeteísta específicamente, no hay motivos serios para favorecer esta solución por sobre la que propone vacíos de verdad. El principal argumento de Priest (2006a, cap. 4) apela a la Exhaustividad de la negación: toda oración que no es verdadera, es falsa, y por eso no puede haber gaps. Sin embargo, esto puede verse como una petición de principio. Porque es claro que un defensor de los vacíos de verdad no va a aceptar esta teoría sobre la negación o la falsedad: para él, la no-verdad y la no-falsedad son compatibles. De este modo, incluso alguien que rechazara la premisa (2) puede, fiel a la tradición filosófica general, seguir sosteniendo el principio de no-contradicción. ${ }^{6}$

El fenómeno de la vaguedad no es el argumento central para el dialeteísmo; de hecho, en el libro central de Priest (2006a) no aparecen consideraciones respecto a eso. Lo que sí aparece es el problema del cambio, que es menos flagrantemente paradójico que el de la vaguedad. ¿En qué sentido es el cambio un problema? Para ver la fuerza intuitiva de esto, podemos imaginar un ejemplo determinado: una copa se cae el suelo y se parte en pedazos. Podemos referirnos al instante mismo en que la copa se rompe y preguntar: ¿es una copa o no lo es? El problema del cambio es el de responder a esta pregunta. Según Priest, si uno acepta el planteo hay cuatro opciones principales:

1. En el instante de cambio, el objeto es una copa.

2. En el instante de cambio, el objeto no es una copa.

\footnotetext{
${ }^{5}$ Williamson (1996) sostiene exactamente esto, agregando que el punto de transición es generalmente desconocido para nosotros.

${ }^{6}$ Podría decir también que la intuición está más del lado de los vacíos de verdad que de las contradicciones verdaderas. Pero no creo que se trate de un buen argumento, como muchos otros que están puramente basados en las sospechosas intuiciones.
} 
3. En el instante de cambio, el objeto es y no es una copa.

4. En el instante de cambio, el objeto ni es ni no es una copa.

El problema con adoptar alguna de las primeras dos opciones es su arbitrariedad: no hay realmente hecho alguno, sea semántico o factual, que determine cuál de esas opciones es correcta. Por eso debemos elegir la tercera o la cuarta opción, donde se mantiene la indiferencia entre la aplicación y la no aplicación del predicado. Ahora bien, como los vacíos de verdad no existen, debemos adoptar la solución dialeteísta, es decir (3).

Este argumento tiene dos puntos problemáticos. En primer lugar, el supuesto de que hay instantes de cambio es discutible. La argumentación a favor de la existencia de tales instantes es muy pobre en el texto de Priest (2006a, p. 162; la traducción es mía):

[Si no hay instantes de cambio], no hay un tiempo en el que el sistema está cambiando. X está antes del cambio. Y está después. Así, en cierto sentido, no hay cambio en el mundo en absoluto, sólo una serie de estados puestos juntos. El universo aparecería más como una secuencia de fotografías, mostradas consecutivamente, que algo en un genuino estado de flujo o cambio.

Sin embargo, más allá de la efectiva metáfora de las fotografías, no resulta claro qué es lo que intenta decir Priest aquí. El cambio puede bien consistir en el paso de un estado a otro, y no requiere necesariamente que haya un instante en donde ese paso "esté ocurriendo"; de ninguna manera eso implica que las cosas no cambien o que el flujo de la realidad sea como un slideshow.

En segundo lugar, aquí sucede algo idéntico a lo que pasaba con la vaguedad. Según Priest, el principal motivo para adoptar (3) y no (4) es, de nuevo, la concepción peculiar de la negación y la falsedad que defiende. Como él mismo admite (2006a, p. 161; la traducción es mía): "La manera más plausible, parece, de atacar este argumento a favor de la existencia de cambios de tipo $\Delta$ [contradictorios] es rechazar el principio de Exhaustividad (si A no es verdadero, entonces $\neg \mathrm{A}$ es verdadero), y así dar lugar a la posibilidad de cambios del tipo $\Gamma$ [vacíos de verdad]". Podemos decir entonces lo mismo que dijimos sobre la vaguedad: que se trata de cierta petición de principio, pues el defensor de los gaps no aceptaría en absoluto el principio de Exhaustividad. 
De esta manera, hemos visto cómo los argumentos metafísicos a favor del dialeteísmo, aunque sugieren interesantes observaciones, están lejos de ser buenos o persuasivos. El principio de no-contradicción puede perfectamente sobrevivir a ellos.

\subsection{Argumentos normativos: leyes}

Las inconsistencias que surgen en el ámbito legal son también otra fuente de argumentación para el dialeteísmo. ${ }^{7}$ Aquí suele distinguirse entre obligaciones inconsistentes y contradicciones legales, siendo las segundas mucho más fuertes para rechazar la no-contradicción.

Tener obligaciones inconsistentes es muy sencillo. Puede pasar, por ejemplo, que uno haga un contrato con $\mathrm{X}$ donde se compromete a hacer $\mathrm{P}$, y también haga un contrato con $\mathrm{Y}$ donde se compromete a no hacer P. De este modo, si ambos contratos son válidos, puedo estar obligado tanto a hacer como a no hacer P. Esto pasa constantemente en los sistemas legales, particularmente cuando las leyes están condicionalizadas. Es común que un individuo, pensando que $\mathrm{A}$ y $\mathrm{B}$ no pueden darse al mismo tiempo, se comprometa a hacer $\mathrm{X}$ sólo si sucede $\mathrm{A}$, y a no hacer $\mathrm{X}$ sólo si sucede $\mathrm{B}$. Pero puede suceder que A y B ocurran a la vez; allí el individuo tendrá obligaciones inconsistentes.

Es poco, sin embargo, lo que estos casos ayudan al dialeteísta. La existencia de obligaciones inconsistentes es plausible, aunque se trata de una contradicción interna: en terminología deóntica, sucede que $\mathrm{Oa \& O} \neg$ a, pero no que $\mathrm{O} a \& \neg \mathrm{O}$. Esto no logra todavía desafiar a la no-contradicción. De hecho, el principal modo de argumentar a favor del principio clásico es buscar la manera de convertir los ejemplos a favor del dialeteísmo en ejemplos de contradicciones internas.

Las contradicciones legales son distintas. Se trata de verdades que resultan de legislaciones existentes, y que llevan la forma de contradicciones externas como "A tiene y no tiene el derecho a P". El surgimiento de estas contradicciones también proviene de casos similares al que mencionamos recién; no hay argumentos elaborados más allá de estos ejemplos y lo que nos parece a primera vista. Por ejemplo: el parlamento de un país establece que

\footnotetext{
${ }^{7}$ De hecho, en Priest (2008), el manual fundamental sobre lógicas no-clásicas, éste es el único argumento no-lógico a favor de los gluts que aparece.
} 
(a) ninguna persona de sexo femenino tiene derecho a votar; y que (b) todos los propietarios de tierras tienen derecho a votar. Ahora, resulta que hay una mujer propietaria de tierras. Entonces, ella tiene y no tiene derecho a votar.

Esto parece ayudar más al dialeteísta, porque se trataría estrictamente de contradicciones verdaderas. Una manera filosóficamente cargada de contestar a esto es decir que la verdad o falsedad no se aplica a oraciones normativas como "A tiene el derecho a P"; la verdad o falsedad se aplicaría a oraciones descriptivas o verificables. Sin embargo, no creo que esta vía sea la adecuada, porque está atada a una división demasiado fuerte entre lo normativo y lo descriptivo, que fue discutida en numerosas ocasiones (magistralmente por Putnam, 2002) y no es aceptada generalmente. Si el argumento contra el dialeteísmo está basado solamente en un concepto tan cargado de lo normativo, entonces no puede servir realmente, pues el dialeteísta es libre de rechazar ese concepto, como lo hacen muchos filósofos contemporáneos.

La segunda manera de responder a esto es proponer que en tales casos la situación es resuelta por alguna cláusula de excepción: por ejemplo, puede haber una ley que diga que entre dos leyes que se contradicen, se tomará en cuenta la ley más nueva, o la de mayor nivel. El problema de esto es que para establecer conclusivamente el punto habría que decir que siempre existen cláusulas de excepción, porque (por ejemplo) las leyes contradictorias pueden haber sido dictadas al mismo tiempo y ser del mismo nivel. Pero la existencia de clásulas de excepción en todos los casos parece implausible. Una manera de lograrlo es considerar que en casos donde no hay una cláusula de excepción, el juez se encarga de resolver este tipo de contradicciones. Priest (2006a, p. 188) observa que esto no ayuda, pues antes de la actuación del juez, la ley era inconsistente, y había por lo tanto una contradicción verdadera; la apelación al juez muestra cómo funciona la dinámica legal para preservar la consistencia, pero no alcanza para negar la inconsistencia contingente de fragmentos legales.

Sin embargo, la observación de Priest no me parece fiel a lo que realmente sucede. En primer lugar, porque una inconsistencia legal que espera a ser resuelta por un juez puede y suele ser vista como un vacío legal: cuando la legislación es contradictoria, consideramos generalmente que la ley no llega a establecer nada concreto al respecto, y será el juez o la corte quien decida cómo llenar este vacío. Si se tratara de un glut, el afectado podría concluir por Simplificación que tiene derecho a $\mathrm{A}$, cuando en realidad no puede 
decir eso razonablemente (pues la ley también dice que no tiene derecho a A). En segundo lugar, porque en estos casos también puede pensarse que el juez, mediante una interpretación abarcativa de la legislación, establece si el afectado tenía o no tenía derecho a A, y no simplemente que está creando ley.

Finalmente, también podría aplicarse una estrategia de internalización de las contradicciones. Por ejemplo, podemos decir que cuando una pieza de legislación genera una contradicción, ya no podemos afirmar que $\mathrm{P}$ tiene derecho ( $\mathrm{y}$ no tiene derecho) a $\mathrm{A}$, sino que según lo que establece la legislación X, P tiene derecho (y no tiene derecho) a A. Esto parece más razonable cuando las contradicciones legales son generadas por leyes distintas: según la ley $\mathrm{X}, \mathrm{P}$ tiene derecho a $\mathrm{A}$, mientras que según la ley $\mathrm{Y}$, P no tiene derecho a A. Este tipo de internalización también podría aplicarse a leyes normales: quizás literalmente sólo sea verdadero que según la legislación X, P tiene derecho o no lo tiene, pero nunca sea verdadero a secas que $\mathrm{P}$ tiene derecho a A. Según Priest, esta estrategia no puede funcionar, pues la internalización de la dimensión legal es anti-intuitiva, como puede notarse en el discurso de los abogados. Es decir, afirmaciones como "A tiene derecho a P" deben ser literalmente verdaderas, y es forzado postular que hay allí un operador implícito que las relativiza a cierta legislación. Sin embargo, creo que hay una dimensión donde esto sucede, y la internalización suele aceptarse: el discurso de ficción. Por ejemplo, si bien solemos afirmar que "Sherlock Holmes usa sombrero", no es porque consideremos que esa oración sea verdadera literalmente (Holmes ni siquiera existe), sino porque aceptamos que "según las historias de Conan Doyle, Sherlock Holmes usa sombrero". Del mismo modo, y esto está más cerca del debate sobre paraconsistencia, muchos relevantistas no dialeteístas aceptan que la historia inconsistente de Priest, "la caja de Sylvan" (Priest 1997), es comprensible y refleja un mundo imposible, sin considerar a la vez que hay contradicciones verdaderas. Es decir, pueden afirmar que "la caja de Sylvan contiene y no contiene un objeto", pero hacen equivaler esa afirmación a que "según el cuento, la caja de Sylvan contiene y no contiene un objeto", lo cual no los compromete con contradicciones verdaderas. De este modo, como la internalización de contradicciones funciona en la ficción, podría funcionar también en la dimensión legal. 


\subsection{Argumentos lógicos}

Sin lugar a dudas, las principales razones para adoptar una postura dialeteísta están relacionadas con las paradojas semánticas. El dialeteísmo es esencialmente una manera más de responder a ellas. Particularmente, la oración del Mentiroso:

(M) M es falsa.

El problema general con esta paradoja es que no puede tener valores estables de verdad. Pues, si es verdadera, es falsa; y si es falsa, es verdadera. Como podemos ver, aquí hay tres factores que provocan la paradoja: el predicado veritativo, la auto-referencia y la lógica clásica. Expondré brevemente las dos soluciones más tradicionales, la clásica y la de vacíos de verdad, para luego pasar específicamente al dialeteísmo. ${ }^{8}$

La tradición tarskiana o clásica atribuye la culpabilidad de esta paradoja, principalmente, a la posesión de un lenguaje semánticamente cerrado: esto es, un lenguaje objeto que exprese sus conceptos semánticos (particularmente, el de Verdad). La solución, entonces, es limitar el predicado veritativo: en el caso original de Tarski, esto se logra desarrollando distintos predicados de Verdad para diferentes niveles de lenguaje, lo cual evita que una oración pueda decir de sí misma que es falsa simpliciter. Cada nivel de lenguaje, $\mathrm{L}_{\mathrm{i}}$, contiene un predicado veritativo $\mathrm{T}_{\mathrm{i}}$ que sólo puede aplicarse a las oraciones del nivel $\mathrm{L}_{\mathrm{i}-1}$. Asimismo, para $\mathrm{L}_{\mathrm{i}}$ se aplicará el predicado veritativo $\mathrm{T}_{\mathrm{i}+1}$.

La dificultad de la solución tarskiana radica, en primer lugar, en resultar demasiado inexpresiva. Teníamos un concepto unívoco de Verdad en nuestro lenguaje natural, y ahora la elucidación nos llevó a una jerarquía de conceptos. No parece haber limitaciones jerárquicas en nuestro uso del concepto de Verdad. Por ejemplo, yo puedo sin dificultades decir 'todas las oraciones de esta página son verdaderas', y es muy comprensible lo que digo. Esta oración, sin embargo, no puede estar en al jerarquía. Por otro lado, como mostró Kripke (1975), a veces la auto-referencia no depende sólo de factores sintácticos, y se pueden obtener paradojas como la del Mentiroso

\footnotetext{
${ }^{8}$ Hay otras innumerables soluciones propuestas a esta paradoja; la elección de la clásica y la de vacíos de verdad se debe simplemente a que son más conocidas y canónicas, pero el debate al respecto es extremadamente largo.
} 
por circunstancias empíricas determinadas. ' Asimismo, tampoco podemos expresar afirmaciones generales respecto de la verdad (por ejemplo, "toda oración es verdadera si y sólo si su negación es falsa"), ya que no tenemos un solo concepto sino múltiples.

La segunda solución tradicional es decir que $\mathrm{M}$ no es ni verdadera ni falsa. Se trata de adoptar gaps, similares a los que antes aparecieron en el debate sobre vaguedad. Esto va de la mano con ciertas creencias instaladas de los lógicos, según las cuales los gaps son mucho más razonables que los gluts (de hecho, el mismo Frege sostuvo que había vacíos de verdad, cuando los nombres propios no tenían denotación). El principal problema de esta posición es que hace surgir paradojas vengativas, como el Mentiroso reforzado:

\section{(MR)MR no es verdadera.}

¿Qué sucede con MR? Sencillo: si no es verdadera ni falsa, por Simplificación no es verdadera. Por lo tanto, es verdadera.

La manera más tradicional de responder a estos problemas es adoptando un principio independiente de lo sintáctico para establecer qué oraciones son indeterminadas o gappy. Particularmente, el requisito de Fundación, que establece que las oraciones con valores de verdad son las que refieren (directa o indirectamente) a hechos no-semánticos. Por ejemplo, si tenemos este conjunto de oraciones:

1. 2 es falsa.

2. 1 es verdadera.

3.

\footnotetext{
${ }^{9}$ El ejemplo famoso de Kripke es el siguiente. Un periodista, Jones, dice: "La mayoría de las afirmaciones que dice Nixon sobre Watergate son falsas." Mientras tanto, Nixon dice: "Todo lo que dice Jones sobre Watergate es falso." En este caso, no hay de por sí una paradoja. Pero una circunstancia empírica puede hacer que la haya: que Nixon haya dicho, hasta ese momento, un número de afirmaciones sobre Watergate tal que la mitad habían sido falsas y la mitad verdaderas; y que Jones sólo haya hecho esa afirmación sobre Watergate.
} 
Ninguna de las dos es fundada, pues refiere a otra oración que asimismo refiere a sí misma, sin jamás lograr pisar tierra firme en algún hecho no-semántico. Algo distinto sucede con las oraciones:

1. 2 es falsa.

2. 3 es verdadera.

3. La hamburguesa es una comida.

Estas tres son fundadas, pues (3) es fundada. De este modo, oraciones como MR resultan indeterminadas, porque no son fundadas. ${ }^{10}$ Esto puede generar ciertas dudas: si MR es indeterminada, entonces de hecho no es verdadera, lo cual la haría verdadera. Sin embargo, la estrategia kripkeana es cortar el nudo desde un principio: si bien podemos decir en cierto sentido (desde el metalenguaje) que MR no es verdadera, desde el lenguaje objeto MR es indeterminada por ser infundada. Esto, desde ya, establece ciertas distinciones entre un lenguaje objeto y un metalenguaje, que obligan a decir a Kripke que el fantasma tarskiano sigue rondando su propuesta. Sucede lo que el dialeteísmo critica: la teoría establece algo (en particular, que MR no es verdadera) que no se puede decir, pero que deberíamos afirmar. Algunos (paradigmáticamente, Maudlin (2006)) proponen una solución en términos pragmáticos: si bien, dada la presencia de vacíos de verdad, muchas oraciones son gappy, algunas de ellas son sin embargo permisibles o asertables. Sin embargo, esto puede verse como otra estrategia ad hoc para exportar los problemas de un lenguaje objeto a un metalenguaje (que esta vez tiene un tinte pragmático): ¿no deberíamos decir que lo que podemos afirmar o asertar con certeza es precisamente verdadero?

Podemos ahora pasar a la versión dialeteísta, que se presenta como una solución a estas paradojas y a los problemas que las anteriores soluciones traían. La propuesta es ahora renunciar a cualquier tipo de jerarquía semántica, y adoptar la clausura: es decir, permitir que los lenguajes lógicos hablen sobre sí mismos y expresen sus propias verdades teóricas. En particular, se liberan las restricciones tarskianas sobre el predicado veritativo, y se adopta un predicado único que puede aplicarse a cualquier oración. También se renuncia a criterios como la fundación para determinar el valor de verdad

${ }^{10}$ Para detalles filosóficos y técnicos, véase Kripke (1975). 
de las oraciones. El precio a pagar es muy alto: la no-contradicción es abandonada, y nos quedamos con una teoría inconsistente, con oraciones verdaderas y falsas. Sin embargo, la lógica subyacente (por su paraconsistencia) permite que haya contradicciones verdaderas sin que esto lleve a la trivialización. Así, habremos de considerar tres valores distintos de verdad: Verdadero, Falso y Ambos. Muchas de las paradojas serán entonces resueltas atribuyendo a las afirmaciones problemáticas el tercer valor de verdad, que a diferencia de lo que sucede en el caso gappy, aquí será un valor designado.

En particular, M y MR serán verdaderas y falsas al mismo tiempo; consecuentemente, $\neg \mathrm{M}$ y $\neg \mathrm{MR}$ también serán verdaderas y falsas. Asimismo, contradicciones como $\mathrm{M} \& \neg \mathrm{M}$, o MR\& $\neg \mathrm{MR}$ también serán verdaderas (pues ambos conyuntos son verdaderas). Esto no impide que estas contradicciones sean también falsas (pues ambos conyuntos son falsos). La renuncia a la no-contradicción, y la aceptación de gluts, no es entonces inocente, pues resulta en una extensa afirmación de contradicciones, y en la declaración de una gran cantidad de oraciones como verdaderas y falsas.

El principal mérito de esta propuesta es lograr un lenguaje semánticamente cerrado. Se supone que el lenguaje natural también lo es, por lo cual esto significa cierto acercamiento hacia él (lo cual siempre es bienvenido). Ciertas barreras expresivas ya no existen, y la estrategia de restricción deja de ser la única viable. Muchas de nuestras intuiciones semánticas pueden ser conservadas (pensemos, por ejemplo, en el esquema T irrestricto), y así el sistema lógico no nos parece, al menos en esos casos, tan lejano a nuestros usos. Lo que nos mostraban las paradojas y los fallidos intentos de solución era precisamente que la consistencia no debe ser mantenida a cualquier precio, y que se trata de un requisito más entre otros.

Aquí, me pronunciaré en contra de la posición dialeteísta. Es muy difícil encontrar críticas internas que puedan derribar esta posición completamente. Mi estrategia será entonces empezar por una crítica externa, para luego analizar algunas objeciones internas a la teoría dialeteísta y ponerlas a la luz de sus propios desiderata.

Un problema general refiere a la plausibilidad de aceptar contradicciones. Como afirma Tennant (2004), los gaps parecen muchas veces más intuitivos y son generalmente utilizados en semántica (Frege, Strawson, etc.) o metafísica, mientras que los gluts gozan de un rechazo generalizado en cualquier teoría filosófica. Por otro lado, muchas de las oraciones que son 
verdaderas y falsas (seguramente, todos los casos más visibles) son casos de oraciones infundadas, por lo que aceptar irrestrictamente las contradicciones puede evitar que hagamos un análisis correcto de cuál es el problema en cuestión. Cada caso debe ser visto por separado y analizado profundamente antes de aceptar la contradicción como un hecho. Asimismo, como ha notado Weir (2004), el dialeteísmo no sirve para falsear teorías. Pues no habilita que, si un argumento es válido, la falsedad de la conclusión derive en que alguna premisa no sea verdadera. Por ejemplo, si nuestra teoría dice $\mathrm{A} \rightarrow \mathrm{B}$ y $\mathrm{A}$, pero encontramos en realidad que $\neg \mathrm{B}$, eso no nos habilita a concluir que nuestra teoría no era verdadera. Finalmente, para muchas teorías de la racionalidad, aceptar contradicciones es irracional; alguien puede creer contradicciones (por algún error epistémico), pero afirmarlas con convicción no parece razonable.

Este problema general es el más importante. Sin embargo, dado que todas estas observaciones externas son respondidas fácilmente mediante la adopción del dialeteísmo y el abandono de la no-contradicción en todos esos ámbitos, iré a algunas críticas más particulares.

En primer lugar, si adoptamos el dialeteísmo, la inconsistencia de la teoría lógica se transmite hacia la metateoría. Por eso, por ejemplo, dada nuestra semántica, para cualquier oración verdadera y falsa a (llamadas "dialeteias" por Priest) se da que Ta\&Fa, pero se dará también que $\neg(\mathrm{T} a$ $\& \mathrm{Fa}$ ) (porque Fa es verdadero y falso a la vez). De esta manera, estamos comprometidos a afirmar que todas las dialeteias no son dialeteias. El teórico dialeteísta se empieza a comprometer entonces con contradicciones metateóricas que no pretendía afirmar, entre las cuales ésta es sólo un ejemplo.

Un segundo problema es la noción de "solamente falso" o "solamente verdadero". Mientras que la tradición clásica considera que afirmar que algo es falso excluye que sea verdadero, para el dialeteísmo esto no sucede. Por lo tanto, necesitamos una manera de afirmar que algo es falso pero no es verdadero. La expresión Fx\& $\neg$ Tx cumpliría ese rol. Sin embargo, pensemos en MR, que es verdadera y falsa. Dado que es verdadera, podemos inferir que no es verdadera. De este modo nos queda que $\mathrm{F}(\mathrm{MR}) \& \neg \mathrm{T}(\mathrm{MR}) \& \mathrm{~T}(\mathrm{MR})$, que afirma algo como 'MR es solamente falsa y verdadera'. Entonces, si bien conceptos como estos son en algún sentido expresables, no nos previenen de inconsistencias: podemos decir que algo es solamente falso, pero quizás luego resulte que también es verdadero. 
La salida propuesta por Priest $(2006 a, 2006 b)$ frente a este problema es pragmática: considerar que la manera de decir conclusivamente que A es simplemente falsa es rechazar A. Afirmar $\neg$ A es todavía compatible con afirmar A; lo que no podemos es afirmar y rechazar a la vez la misma oración. Sin embargo, además de la reforma radical que esto significa para cualquier teoría de la aserción, aquí volvemos a lo que el dialeteísta criticaba: la exportación de problemas semánticos a la dimensión pragmática. Y por otro lado, no estamos libres de paradojas en ese plano. Por ejemplo:

(MA) MA no es asertable.

Si MA es verdadera, entonces es asertable (pues todo lo verdadero es asertable); pero que sea verdadera implica también que no es asertable. Por otro lado, si MA es falsa, entonces es asertable; pero si es asertable, es verdadera, lo cual a su vez implica que no es asertable. Por lo tanto, MA es asertable y no es asertable.

En tercer lugar, la noción de teorema se corre de la tradicional: en particular, todo teorema es falso bajo alguna interpretación (particularmente, la interpretación que asigna Ambos a todo símbolo proposicional). De esta manera, los teoremas son siempre verdaderos y a veces también falsos.

Finalmente, algunas verdades lógicas que el dialeteísta parece no aceptar son teoremas en su sistema y por lo tanto son aceptadas. Eso sucede en particular con el Principio de no-contradicción, es decir, la fórmula $\neg($ A\& $\neg$ A).

De esta manera, el dialeteísta no está en un lugar mucho más cómodo que el clásico respecto a sus compromisos. ¿Cómo puede defenderse una teoría sobre las paradojas que supone que la solución propuesta (por ejemplo, que $\mathrm{M}$ es una dialeteia) es y no es verdadera? ¿Cómo se entiende el rechazo de la no-contradicción si la fórmula que lo expresa es aceptada como teorema? ¿De qué manera podemos considerar al dialeteísmo como una teoría estable en el plano pragmático si habrá oraciones asertables y no asertables? ¿Cómo podemos afirmar que tenemos la mayor expresividad posible si nuestro concepto de "solamente falso" no excluye el de "verdadero"? Todo eso debe verse (a diferencia de lo que diría Priest) como indeseable, y no puede salvarse con la mera aceptación de contradicciones y la caracterización de los adversarios como dogmáticos. Parece que el dialeteísta necesitaba alguna tierra firme para afirmar conclusivamente 
sus propios principios o soluciones; pero ha terminado afirmando no sólo sus propuestas radicales, sino también los principios de sus adversarios. Tampoco es evidente que esté libre de paradojas, o que haya logrado definitivamente la expresividad absoluta. Todo esto se suma al gran problema general que significa la aceptación de contradicciones.

\section{Paraconsistencia doxástica}

Expuestas ya las dificultades del dialeteísmo, parece que dar bienvenida a las contradicciones implica no sólo el abandono de un principio central sino también la adopción de una actitud contradictoria frente a los propios enunciados metateóricos. La paraconsistencia, sin embargo, puede separarse de esto: como señalé antes, una lógica es paraconsistente cuando A\& $\neg$ A no implican B, y no necesariamente debe aceptar contradicciones como verdaderas a secas.

Sin embargo, la mayoría de las lógicas paraconsistentes poseen semánticas en las cuales los modelos aceptan inconsistencias (el caso más conocido en este marco es la lógica relevante). La manera tradicional de acomodar esto es decir que esos modelos no representan estados reales de cosas, sino estados doxásticos: maneras en como el mundo no puede ser, pero sin embargo pueden ser creadas, imaginadas, creídas o incluso deseadas. Esta posición, que me parece la adecuada, fue sostenida contemporáneamente por Restall (1997), entre otros lógicos relevantistas.

El caso más ilustrativo es la ficción de Priest "la caja de Sylvan": un cuento perfectamente comprensible que recrea una situación contradictoria; esa historia, asimismo, podemos imaginarla. Del mismo modo puede admitirse la posibilidad de tener creencias inconsistentes sin ser irracionales: podemos, por ejemplo, creer que nuestra habitación mide $2 \mathrm{~m}$ de ancho y 3 de largo, pero al mismo tiempo que tiene $9 \mathrm{~m}^{2}$. Aquello que es creído en este caso es imposible y contradictorio. También podemos creer, como hace Priest, que el conjunto de Russell pertenece y no pertenece a sí mismo, o que en casos intermedios un objeto puede ser y no ser azul.

Es cierto, sin embargo, que no podemos hacernos una verdadera imagen de una situación contradictoria, y que nuestra representación de ella es confusa (salvo que consideremos, como Priest, que los casos intermedios o dinámicos son contradictorios). Pero no es la única representación 
confusa de la cual no tenemos una imagen: pensemos en enunciados puramente teóricos a los que sin embargo atribuimos sentido ("la monarquía es una forma de gobierno surgida de lo Absoluto").

Así podemos también ver intuitivamente por qué, en un sentido (el relevantista), argumentos como el Ex falso pueden fallar cuando no hay contenido en común entre la premisa contradictoria y la conclusión (en otras palabras, cuando no hay relevancia). Con sólo concebir una contradicción no trivializante (por ejemplo, que M sea verdadera y falsa, o que la caja de Sylvan contenga y no contenga un objeto), ya es suficiente para ver que en esos casos en las premisas contradictorias se dan, pero la conclusión no.

Esto también nos ayuda a diferenciar oraciones como 'si logro la cuadratura del círculo, me transformo en pato', de otras como 'si logro la cuadratura del círculo, me hago famoso', a partir de una lectura relevantista del condicional: en la situación imposible donde logro la cuadratura del círculo, seguramente me haría famoso, pero no me convertiría en pato.

En conclusión, y respecto a las contradicciones antes vistas, la estrategia de la paraconsistencia doxástica (que yo defiendo) es internalizar las contradicciones. Cuando ellas surgen, no diremos que que A\& $\neg$ A (elíjase la contradicción que uno prefiera), sino que en la situación $X$ (que puede ser producto de creencias, teorías, ficciones, sueños, deseos, etc.), A\& $\neg \mathrm{A}$. La consideración misma de esas situaciones, inconsistentes pero no triviales, es lo que nos previene de aceptar irrestrictamente el Ex falso y nos motiva para la paraconsistencia. Así evitamos la afirmación de contradicciones, damos a la paraconsistencia un motivo razonable y acomodamos en la teoría la posibilidad de tener creencias contradictorias racionalmente.

Un argumento de pendiente resbaladiza (Priest, entre otros, suele plantearlo) nos puede llevar a pasar de la aceptación de teorías o estados de cosas inconsistentes a la aceptación de contradicciones verdaderas. Sin embargo, creo que es razonable poner un límite antes de renunciar a la no-contradicción: las teorías o creencias contradictorias no pueden ser verdaderas. Hace falta responderle al idealista que plantea que, después de todo, si el mundo está construido por nuestras teorías, debería poder ser contradictorio. Mi respuesta es sencilla: más allá de los debates sobre realismo o idealismo, debemos admitir que podemos estar equivocados, y lo estamos particularmente cuando consideramos que una teoría contradictoria puede ser verdadera. 


\section{Bibliografía}

Batens, Diderik, "Against Global Paraconsistency", Studies in Soviet Thought 39, 1990, pp. 209-229.

Berto, Francesco, How to Sell a Contradiction, College Publications, Londres. 2007.

Kripke, Saul, "Outline of a Theory of Truth", Journal of Philosophy 72, 1975, pp. 690-716.

Kroon, Frederick, "Realism and Dialetheism", The Law of Non-Contradiction. Ed. Graham Priest, J. C. Beall and Bradley Armour-Garb, Oxford, Oxford University Press, 2004, pp. 245-263.

Lewis, David, "Logic for Equivocators", Noûs 16, 3, 1982, pp. 431-441.

Lewis, David, "Letter to G. Priest", The Law of Non-Contradiction, Ed. Graham Priest, J. C. Beall and Bradley Armour-Garb, Oxford, Oxford University Press, 2004, pp. 176-177.

Maudlin, Tim, Truth and Paradox, Oxford University Press, Oxford, 2006.

Mares, Edwin, Relevant Logic: a Philosophical interpretation, Cambridge University Press, Cambridge, 2001.

Neurath, O. (1932), "Proposiciones protocolares", El positivismo lógico, Ed. Alfred Ayer, Fondo de Cultura Económica, México DF, 1977.

Putnam, Hilary, The Collapse of the Fact/Value Dichotomy and Other Essays, Harvard University Press, Cambridge MA, 2002.

Priest, Graham, In contradiction: 2nd edition, Oxford Clarendon Press, Oxford, 2006 (2006a).

Priest, Graham, Doubt truth to be a Liar, Oxford University Press, Oxford. 2006 (2006b).

Priest, Graham. "What's so bad about contradictions", The Law of Non-Contradiction, Ed. Graham Priest, J. C. Beall and Bradley Armour-Garb. Oxford University Press, Oxford, 2004, pp. 23-38.

Priest, Graham, Introduction to non-classical logic: from if to is, Oxford University Press, Oxford, 2008.

Priest, Graham, "Sylvan's Box", Notre Dame Journal of Formal Logic 38, 4, 1997, pp. 573-582.

Quine, Willard van Orman, Philosophy of Logic, Prentice Hall, New Jersey, 1970.

Restall, Greg, "Ways things can't be", Notre Dame Journal Of Symbolic Logic 38, 4, 1997, pp. 583-596. 
Restall, Greg, "Carnap's Tolerance, Language Change and Logical Pluralism", Journal of Philosophy 99, 2001, pp. 426-443.

Stalnaker, Robert, Ways a World Might Be: Metaphysical and Anti-Metaphysical Essays, Oxford University Press, Oxford, 2003.

Tennant, Neil, "An anti-realist critique of Dialetheism", The Law of Non-Contradiction, Ed. Graham Priest, J. C. Beall and Bradley Armour-Garb, Oxford University Press, Oxford, 2004, pp. 355-384.

Varzi, Achille, "On Logical Relativity", Philosophical Issues 10, 2004, pp. 197-219.

Weir, Alan, "There are no true contradictions", The Law of Non-Contradiction, Ed. Graham Priest, J. C. Beall and Bradley Armour-Garb, Oxford University Press, Oxford, pp. 385-417.

Woods, John, Paradox and paraconsistency, Cambridge University Press, Cambridge, 2003.

Recibido 7/2014; aceptado 10/2014 